\title{
Situación de la formación profesional y la organización gremial del trabajo social en Honduras
}

\author{
María Victoria Ponce Mendoza, Deisi Noemí Gallardo López
}

\section{RESUMEN}

El objeto de la investigación fue la contemporaneidad de la formación académica del profesional de trabajo social en el contexto de las reformas universitarias y de la situación sociopolítica del país, basándose en dos grandes dimensiones: el proceso de formación académica del profesional de trabajo social en la UNAH, con base en el plan de estudios aprobado en 1999, vigente aún en el periodo estudiado, 20112012 y la organización gremial del trabajo social en Honduras.

El proceso de investigación fue desarrollado desde el paradigma interpretativo crítico, con enfoque metodológico cualitativo, fenomenológico con procedimiento sistémico; las técnicas de investigación utilizadas fueron la entrevista a profundidad, entrevista semiestructurada, grupos focales y análisis de contenido.

Entre los principales resultados se encontró que en el plan de estudios vigente no está claramente conceptualizada la articulación metodológica de los cuatro bloques de la formación académica; asimismo, que hay ausencia en el planteamiento relacionado con la búsqueda del compromiso ético-político de la profesión, se evidencia la falta de conceptualización y fragmentación desde una perspectiva teórica-metodológica de y entre las prácticas intermedias y con la práctica final.

En conclusión, el plan de estudio de la Carrera de Trabajo Social y su respectivo perfil profesional requiere ser actualizado considerando la complejidad de la cuestión social, lo cual hace necesario implementar el modelo educativo de la UNAH que incorpore la investigación como un quehacer fundamental en el proceso de formación y de la labor docente de la Carrera de Trabajo Social. En cuanto a la organización gremial, existe una desvinculación entre el Colegio de Profesionales de Trabajo Social de Honduras y la academia; razón por la cual hacen faltan propuestas y posicionamientos por parte del gremio ante la realidad nacional, situación que disminuye su beligerancia ante la sociedad hondureña.

Palabras clave: formación académica, trabajo social, proceso teórico-metodológico,

${ }^{1}$ Universidad Nacional Autónoma de Honduras. Facultad de Ciencias Sociales, Departamento de Trabajo

Social. Correo electrónico: poncevictoria@gmail.com; deisiapre@gmail.com 
cuestión social, compromiso ético-político, organización gremial.

\section{ABSTRACT}

The objective of the investigation was the contemporaneity of the Academical Formation of the Social Work Professionals, according to the University Reforms and the Social Political Situation of our Country, based on the study of two big dimensions, one was the process of the Academical formation of the Social Workers of the UNAH, and the Study Plan adopted by the Career in 1999, which was used up to 2012; and the other one was the Social Worker Union in Honduras. The process of the investigation was developed from an interpretative and critical paradigm, under a methodological, qualitative and phenomenological approach with a systemic procedure. The investigation techniques applied were a very well structured interview, a semi-structured interview, focal groups, and the analysis of the content.

Among the main results, we found that the four blocks of the Academical formation are not clear enough conceptualized or articulated methodologically, talking. There is a lack of statements related to the Ethic -political compromise of the profession; it is also evident the lack of a conceptualization and a fragmentation from the perspective of a methodological - theory, of, and, among the intermediate ant final Practicums. In conclusion, the Study Plan of the Social Work Career and its Professional Profile needs to be updated, taking into consideration the complexity of the social issues. So, it is needed to implement the New Educative Model of the UNAH, incorporating the investigation, as a main task, along the formation process.

Talking about the Union Organization, there is not any link between the Union of The Social Professional Workers of Honduras and the School of Social Work of the UNAH, so it is mandatory to establish some proposals by the Union of the Social Worker side, before the real situation of the Honduran Society that diminishes his belligerence before it.

Key words: academical formation; social work; theoretical-methodological process, social issues, ethic-politic compromise, and gremial organization or union organization.

\section{INTRODUCCIÓN}

En este artículo se dan a conocer los principales resultados logrados en el proceso de investigación sobre el tema que aborda el estado de situación de la formación profesional y organización gremial del trabajo social en el contexto hondureño, 
durante el período comprendido entre los años 2011-2012.

El objeto de la investigación fue la contemporaneidad de la formación académica profesional del trabajo social en la UNAH, en el contexto de las reformas universitarias y de la situación sociopolítica del país, analizando el proceso de formación académica del profesional con base al plan de estudios aprobado en 1999, que aún está vigente en la actualidad; asimismo, se analizó el funcionamiento de la organización gremial del trabajo social en Honduras.

La principal justificación para realizar el estudio fue la conveniencia de determinar las características generales de la organización gremial, las percepciones sobre los desafíos y estrategias con que cuenta para visibilizar el protagonismo que los profesionales egresados de la Carrera de Trabajo Social de la UNAH tienen en las diferentes áreas en que implementan las políticas públicas; asimismo, identificar aspectos por fortalecer y áreas que potenciar tanto en el trabajo gremial en sí, como en la interrelación que se establece con la carrera universitaria y la retroalimentación que se da en este proceso para favorecer los cambios en el diseño y proceso de desarrollo curricular.

De igual manera, los resultados del estudio permitirán a la Carrera de Trabajo Social ponerse a tono con las nuevas tendencias y lineamientos educativos para enfrentar los desafíos humanos-sociales que se presentan en la realidad actual hondureña; en este sentido, los resultados del estudio también servirán de base para realizar los cambios correspondientes en el proyecto curricular de dicha carrera, para que propicie la acreditación de la formación de profesionales asumiendo posturas analíticas, reflexivas, propositivas y proactivas, facilitando de este modo los procesos de cambio a favor de la calidad de vida de la población hondureña.

El proceso de investigación se desarrolló desde el paradigma interpretativo crítico, con enfoque metodológico cualitativo, con procedimiento sistémico, utilizando técnicas de investigación como la entrevista a profundidad semiestructurada, grupos focales y análisis de contenido. Espacialmente, se cubrieron cuatro regionales, incluyendo las ciudades de Tegucigalpa, Choluteca, San Pedro Sula y La Ceiba. En este contexto, por un lado se indagó sobre el proceso de formación profesional de la Carrera de Trabajo Social y sobre las percepciones acerca de la experiencia lograda en diferentes áreas de intervención profesional en la validación del perfil curricular, en espacios públicos, como en las organizaciones no gubernamentales, tomando en cuenta el contexto sociopolítico nacional en el marco del modelo neoliberal y de las reformas universitarias; por otro lado, se investigó la funcionalidad de la organización gremial del trabajo social y su vinculación con la 
sociedad hondureña.

Al analizar el proceso de la formación académica del trabajo social, fueron consideradas las dimensiones ético-político y la teórica metodológica, puesto que los objetivos de la investigación en esta dimensión de estudio fueron los siguientes:

1. Identificar las características del proceso de la formación profesional en trabajo social en Honduras, desde las mediaciones estructurales, coyunturales, universitarias y extrauniversitarias, constitutivas de las carreras de trabajo social o escuelas formadoras.

2. Reconocer las características del perfil de formación profesional con especial énfasis en las dimensiones ético- político y teórica-metodológico que muestra el plan de estudios de la Carrera de Trabajo Social, para ser consecuente con las exigencias de un proyecto de sociedad que busque la equidad y lajusticia social.

3. Analizar los ejes curriculares básicos del plan de estudios y su relación con las dimensiones teórica- metodológica y ética-política en el proceso de formación profesional.

\section{METODOLOGÍA}

El proceso de investigación fue desarrollado desde el paradigma interpretativo crítico, dando al estudio un enfoque metodológico cualitativo, cuyo propósito fue la comprensión y construcción de conocimiento acerca de nuestro objeto de investigación, a fin de dar una visión holística, amplia, integrada y contextualizada tanto a nivel universitario como extrauniversitario, permitiendo esto clarificar, sintetizar, comparar, analizar y reflexionar acerca de la experiencia subjetiva de los actores clave con relación al objeto de estudio. Para el análisis de la información se utilizaron cuadros matrices y la técnica de triangulación.

La población y muestra del estudio estuvo constituida por docentes y estudiantes de la Carrera de Trabajo Social, dirigentes y exdirigentes del Colegio de Profesionales de Trabajo Social de Honduras y profesionales de trabajo social que laboran en diferentes áreas y en instituciones públicas y privadas en cuatro ciudades importantes del país: Tegucigalpa, la capital de la república, en el departamento de Francisco Morazán, en zona central del país; San Pedro Sula, capital industrial, en el departamento de Cortés; La Ceiba, en el departamento de Atlántida y en la ciudad de Choluteca, en el departamento de Choluteca, zona sur. 
En una primera etapa se identificaron y seleccionaron las técnicas apropiadas para la recolección de datos e información, se realizaron pruebas piloto de los instrumentos y se ajustaron previamente para su aplicación. Dada la naturaleza del estudio, se utilizaron cuatro técnicas con sus respectivos instrumentos para trabajar con los diferentes actores clave: entrevistas a profundidad, grupos focales, análisis documental y el cuestionario. La técnica de grupos focales fue aplicada a ocho sectores involucrados en las cuatro ciudades importantes que formaron parte de la población y muestra del estudio.

En la segunda etapa se realizó la recolección de datos e información de las fuentes primarias y secundarias, las entrevistas a profundidad fueron dirigidas a representantes de las autoridades universitarias, a nivel de la Dirección de Vinculación Universidad-Sociedad, Decanatura de la Facultad de Ciencias Sociales, Jefatura del Departamento y Coordinación de la Carrera de Trabajo Social, así como a la Dirección y docentes de la Maestría Latinoamericana de Trabajo Social.

En una tercera etapa se incorporaron sugerencias producto de la participación en el VI Congreso de Investigación Científica de la DICU, de profesionales de la Maestría Latinoamericana de Trabajo Social y del Departamento de Sociología de la Facultad de Ciencias Sociales; igualmente, las sugerencias de las jornadas de socialización con los sectores involucrados para la validación y retroalimentación del informe final.

\section{LAFORMACIÓN PROFESIONAL DEL TRABAJO SOCIAL EN HONDURAS}

Para el estudio del perfil profesional actual de la Carrera de Trabajo Social vigente desde 1999, se tomaron en cuenta sus diferentes componentes, iniciando por relacionar los conocimientos, habilidades y destrezas de mayor relevancia y prioridad planteadas en el mismo.

Al analizar la formulación del perfil profesional en lo que se refiere a los conocimientos, habilidades y destrezas establecidas en el mismo, se logra determinar que, de acuerdo a las tendencias, se trata de un perfil eminentemente tecnocrático, ya que enfatiza los conocimientos, habilidades y destrezas en la elaboración de investigaciones para la planificación y la administración de planes y programas de desarrollo según su calificación.

Es decir, que el diseño del perfil profesional está influenciado fuertemente por las tendencias teóricas del interaccionismo simbólico y otras teorías posparsonianas; corriente de pensamiento que se sustenta en la filosofía pragmática, vinculada al orden social que tiene una acción y una dimensión de racionalidad e ideal normativo. 
Esta influencia se explica dado que el plan de estudios vigente se elaboró en la década de los años 90 , con teorías que sirven de fundamento al neoliberalismo en la definición de las políticas públicas y en las que se enfatiza que los problemas de la distribución de recursos se resolverán por la interacción de los individuos y por el equilibrio natural que genera dicha interacción.

A esas tendencias teóricas se le han denominado tecnicistas, lo cual también se refleja en el perfil profesional, basado fundamentalmente en el desarrollo de un conjunto de instrumentos que permitan mejorar la gestión social, para lo cual se requiere hacer investigaciones empíricas para la comprensión de los fenómenos sociales y la implementación de proyectos, donde el profesional tiene que administrar eficientemente los recursos y dar respuesta a la demanda de acuerdo a lo establecido en el plan.

Si bien es cierto que la Carrera de Trabajo Social ha respondido a la demanda del perfil profesional que enfatiza, precisamente, una formación de corte tecnocrático - tal como lo ha exigido la demanda de las empresas públicas y privadas en el modelo neoliberal- , los empleadores consideran que aún falta desarrollar más esas nuevas capacidades, por ejemplo, en el conocimiento, habilidad y destreza para trabajar en la sistematización de experiencias, temática que aparece en el perfil profesional del trabajo social, pero que no está contemplada como asignatura en el plan de estudios vigente.

Los empleadores también solicitan capacidades llamadas genéricas, que de acuerdo a la Agencia Nacional de Evaluación de la Calidad y Acreditación de España, son los atributos que debe tener un graduado universitario con independencia de su titulación. Estas capacidades genéricas las organizó el Proyecto Tuning-Latinoamérica en capacidades instrumentales, personales y sistémicas (ver tabla 1).

El modelo pedagógico de la UNAH apela a la profesión y disciplina para asumir una perspectiva de análisis: "Crítica y reflexiva, porque ello implica un compromiso directo y explícito de los sujetos por trasformar las actuales relaciones sociales. Señalando, además, que la teoría crítica reflexiva permite aprehender la relación dialéctica inherente e interdependiente de un pensamiento político-filosófico, capaz de generar conciencia social, transformadora y emancipadora en el contexto de la justicia, la democracia y la libertad" (UNAH, 2009). 
Tabla 1. Proyecto Alfa Tuning América Latina: capacidades genéricas

\begin{tabular}{|c|c|c|}
\hline Instrumentales & Personales & Sistemáticas \\
\hline $\begin{array}{l}\text { - Capacidad de abstracción, } \\
\text { análisis y síntesis } \\
\text { - Capacidad de comunicación } \\
\text { oral y escrita } \\
\text { - Capacidad de comunicación en } \\
\text { un segundo idioma } \\
\text { - Habilidades en el uso de las } \\
\text { tecnologías de la información y } \\
\text { de la comunicación } \\
\text { - Capacidad para organizar y } \\
\text { planificar el tiempo } \\
\text { - Capacidad de aplicar los } \\
\text { conocimientos en la práctica } \\
\text { - Conocimientos sobre el área de } \\
\text { estudio y la profesión } \\
\text { - Capacidad de investigación } \\
\text { - Habilidades para buscar, } \\
\text { procesar y analizar información } \\
\text { procedente de fuentes diversas } \\
\text { - Capacidad para formular y } \\
\text { gestionar proyectos } \\
\text { - Capacidad para tomar } \\
\text { decisiones }\end{array}$ & $\begin{array}{l}\text { - Capacidad de trabajo en } \\
\text { equipo } \\
\text { - Habillidades } \\
\text { interpersonales } \\
\text { - Habilidad para trabajar } \\
\text { en contextos } \\
\text { internacionales } \\
\text { - Valoración y respeto por } \\
\text { la diversidad y } \\
\text { multiculturalidad } \\
\text { - Capacidad crítica y } \\
\text { autocrítica } \\
\text { - Compromiso ético } \\
\text { - Compromiso con la } \\
\text { calidad }\end{array}$ & $\begin{array}{l}\text { - Habilidad para trabajar en } \\
\text { forma autónoma } \\
\text { - Responsabilidad social y } \\
\text { compromiso ciudadano } \\
\text { - Capacidad de aprender y } \\
\text { actualizarse } \\
\text { permanentemente } \\
\text { - Capacidad para actuar en } \\
\text { nuevas situaciones } \\
\text { - Capacidad creativa } \\
\text { - Capacidad para } \\
\text { identificar, plantear y } \\
\text { resolver problemas } \\
\text { - Capacidad de motivar y } \\
\text { conducir hacia metas } \\
\text { comunes } \\
\text { - Compromiso con la } \\
\text { preservación del medio } \\
\text { ambiente } \\
\text { - Compromiso con su } \\
\text { medio sociocultural }\end{array}$ \\
\hline
\end{tabular}

Fuente: Elaboración propia con base a Proyecto Tuning-Latinoamerica.

Al analizar el perfil profesional se visualizó que los principales elementos éticos propuestos por el modelo educativo de la UNAH no están incorporados, cuando este señala que todas las carreras de la UNAH deben contemplar la ética y la bioética, como una de las líneas curriculares fundamentales, abordando asuntos como el bien común, la responsabilidad, la problemática de la corrupción, los principios de transparencia y rendición de cuentas, las implicaciones éticas y legales de la profesión, los derechos humanos particulares y universales, enfatizando el valor universal de la vida y la dignidad inherente a todo ser humano (UNAH, 2009).

Al analizar el peso y composición de los bloques de formación en el plan de estudio, se muestra que el bloque de formación y eje articulador teórico-metodológico no está conceptualizado y no se determinan los mecanismos para lograr la articulación de las prácticas intermedias y del personal docente en cuanto a la planificación y organización del proceso de formación en la Carrera de Trabajo Social, para que se 
puedan articular experiencias y saberes de cada una de ellas en el ejercicio de la docencia, encaminado esto al logro de los objetivos asumidos en el perfil profesional.

\section{ORGANIZACIÓN GREMIAL DEL PROFESIONAL DE TRABAJO SOCIAL EN HONDURAS}

La organización gremial de los profesionales del trabajo social surgió con mucho entusiasmo en un contexto en el que se asumió un compromiso de proyecto político vinculado con los grupos de población que demandaban justicia social, especialmente en cuanto a la posesión de la tierra, es decir, para los campesinos sin tierra y los pobladores de origen rural que habían migrado a la ciudad. Estas circunstancias agudizaban las contradicciones y la conflictividad social ante el Estado que, en la década de los años 70, impulsaba procesos de industrialización por sustitución de importaciones e impulsaba políticas sociales que requerían a profesionales del trabajo social para la implementación de las mismas, lo que posibilitó la validación profesional para licenciatura en Trabajo Social.

Sin embargo, con el paso de los años, especialmente a partir de la década de los 90 , cuando la economía de mercado logra su hegemonía mundial dictaminando las nuevas reglas del juego para América Latina, mediante el llamado Consenso de Washington, se llevan a cabo medidas conducentes al achicamiento del Estado y a la reducción de su papel planificador e impulsor de políticas públicas, lo que da como resultado la privatización de la mayor parte de los servicios de asistencia social, orientados especialmente a la población femenina e infantil, lo provocó que el Estado trasladara su responsabilidad a las organizaciones privadas de desarrollo, logrando así en alguna medida, disminuir la afiliación de los egresados al gremio profesional, puesto que para laborar con estas organizaciones no se solicitaba como un requisito obligatorio la colegiación gremial y normalmente los profesionales se movilizaban a realizar su labor en zonas alejadas del nivel central, sin ningún contacto o vinculación con la organización gremial.

Al relacionar las percepciones de los profesionales del trabajo social en las diferentes regionales del país, se destaca la insatisfacción existente entre los agremiados que aún mantienen vínculos organizativos entre ellos; pero, la estructura organizativa y funcionamiento de la organización gremial no posibilita ni facilita la comunicación efectiva, pese a la disponibilidad y facilidad que brinda la tecnología moderna para la resolución de problemas de tipo administrativo y legal, que se deben enfrentar y resolver para proteger los derechos establecidos en el estatuto profesional. 
Es interesante destacar que inicialmente la academia se vinculó a puestos de dirección de la organización gremial, incorporándose con mucho compromiso y entusiasmo, sin embargo, en los últimos años se hace evidente el distanciamiento del personal que labora en la academia con la organización gremial. Asimismo, se destaca la falta de posicionamiento de los profesionales como gremio organizado ante la problemática social, económica, política y cultural; particularmente ante los hechos acaecidos durante y después del golpe de Estado del 28 de junio de 2009.

En consecuencia, hay retos y desafíos que el gremio profesional debe resolver a mediano y corto plazo, a fin de fortalecer su estructura organizativa y adecuado funcionamiento, entre los cuales se pueden mencionar los siguientes:

1. Considerar las percepciones y sugerencias de las diferentes filiales y de actores participantes para convertir a la organización en una verdadera entidad jurídicalegal, que permita el establecimiento de redes profesionales representativas del gremio a nivel de las diferentes regionales, con vínculos efectivos de comunicación, de propuesta y de gestión.

2. Formular la planificación estratégica que incorpore entre sus diferentes áreas la capacitación y actualización profesional en coordinación con la academia. De igual manera, impulsar el área de promoción para el posicionamiento de la profesión, tanto a nivel de los mismos agremiados, como ante las diferentes instituciones públicas y organizaciones privadas de desarrollo a nivel nacional.

3. Estrechar lazos de coordinación y de trabajo con la academia en el nivel de grado y postgrado, con personal docente y estudiantil, para consolidar procesos de fortalecimiento organizacional e institucional para ambas instancias.

\section{RESULTADOS}

1. Se evidenció que en el plan de estudio vigente en la Carrera de Trabajo Social no hay claridad conceptual con relación a la articulación metodológica de los cuatro bloques de formación académica, aspecto que limita la coordinación y armonización de contenidos de las asignaturas a nivel horizontal y vertical, lo que dificulta a su vez, el fortalecimiento de la capacidad analítica, reflexiva y crítica por parte de los estudiantes en el abordaje de la "cuestión social" del campo profesional.

2. En el plan de estudio de la Carrera de Trabajo Social está ausente el proyecto relacionado con la búsqueda del compromiso ético-político de la profesión, que esté en correspondencia con las demandas de los grupos de población.

3. La ausencia de conceptualización y de vinculación desde una perspectiva teórica-metodológica de diferentes prácticas intermedias y de la práctica 
profesional que se lleva a cabo al final de la carrera, es un factor que dificulta la visualización del paradigma epistemológico que está orientando 0 enriqueciendo la acción social de los profesionales del trabajo social.

\section{CONCLUSIONES}

1. El contexto extrauniversitario del proceso de formación del trabajo social en Honduras, ha estado mediado estructural y coyunturalmente por el enfoque neoclásico, impuesto por los teóricos del neoliberalismo, cuyo fundamento está en la defensa de la eficacia del mercado como mecanismo de asignación óptima de los recursos y con políticas públicas que están focalizadas en los más pobres de los pobres y basadas en el enfoque de manejo del riesgo social.

2. La formación profesional del trabajo social ha tenido una clara tendencia funcional-estructuralista al incorporar conceptos en armonía con el sistema neoliberal hegemónico, que plantea como funciones del profesional de trabajo social en el abordaje de la cuestión social, la administración de recursos con eficiencia y la eficacia planteados desde la visión empresarial.

3. El surgimiento de nuevos actores sociales, tales como los defensores de los derechos humanos, los ambientalistas, las organizaciones de mujeres, organizaciones de jóvenes, organizaciones de pueblos indígenas y las organizaciones comunitarias, han adquirido protagonismo a nivel regional 0 nacional, llevándoles al planteamiento de demandas desde sus particularidades, que deben ser atendidas por profesionales del trabajo social como parte de su ámbito de acción en la esfera de la cuestión social.

4. Las instituciones del Estado se vuelven inoperantes por la reducción del gasto público en la implementación de las políticas sociales ante la incapacidad de dar respuesta a la demanda de la población en la exigibilidad de sus derechos, por lo cual los profesionales de Trabajo Social, enfrentan serias dificultades administrativas para llevar a cabo la intervención social por lo que, en su afán de dar respuesta a las necesidades de la población, están llevando a cabo un trabajo basado más en la gestión estrictamente personal, que en los recursos asignados por las diferentes dependencias del Estado en las que se desempeñan.

5. En los conocimientos, habilidades y destrezas establecidas en el perfil profesional del trabajo social, se logró evidenciar que se trata de un perfil orientado eminentemente a la formación de tipo tecnocrática o tecnicista, enfatizando los conocimientos, habilidades y destrezas en la elaboración de investigaciones para la planificación y la administración de planes y programas llamados de desarrollo congruentes con los objetivos del plan de estudios de la carrera, pero no muestran que responde a un proyecto político definido en 
función de las grandes mayorías de la sociedad hondureña.

6. Tanto las entidades públicas, como de organismos no gubernamentales, coinciden en afirmar que la carrera necesita fortalecer conocimientos, habilidades y destrezas en los profesionales egresados del trabajo social en aspectos como: investigación, planificación, sistematización de experiencias, gerencia por resultados, administración de presupuestos y redacción de informes entre otras.

7. En el análisis del perfil profesional y su énfasis en la dimensión ética- política, teórica- metodológica, uno de los obstáculos encontrados es el de sostener una propuesta con una tendencia teórica eminentemente tecnicista, dando mayor énfasis y prioridad a los conocimientos, habilidades y destrezas encaminados a la elaboración de investigaciones para la planificación y la administración de planes y programas de desarrollo, pero no para la reflexión crítica, análisis, propuesta y producción de conocimiento científico de acuerdo a la demanda que la realidad hondureña actual exige y consecuente con un proyecto de sociedad que se opone a la naturalización de la desigualdad social.

8. Los ejes curriculares del plan de estudios de la carrera, en consonancia con el perfil profesional del trabajo social, no están en correspondencia con los desafíos actuales de la sociedad hondureña, por lo que se puede afirmar que existe una desarticulación entre los cuatro ejes curriculares o bloques de asignatura.

9. El eje curricular teórico-metodológico considerado de acuerdo al plan de estudios como el eje articulador del proceso de formación profesional, aunque cuenta con el mayor peso en unidades valorativas, presenta varias limitaciones al no estar conceptualizado ni señaladas las estrategias de articulación en el proceso de formación para intervenir en la cuestión social, tal parece que de acuerdo a las percepciones del personal docente, no se está logrando totalmente la finalidad articuladora.

10. Los contenidos de las asignaturas del eje curricular teórico-metodológico no muestran la existencia de una guía secuencial que exponga la tendencia teórica que responde 0 da origen a los métodos tradicionales en la intervención de la cuestión social.

11. Tanto los ejes curriculares del proceso de formación como el perfil profesional definido en el plan de estudios de la Carrera de Trabajo Social necesitan ser actualizados para ofrecer una respuesta más eficiente a las demandas sociales y a la conformación de un proyecto ético -político que busque la formación de profesionales de trabajo social con capacidades personales, sistémicas e instrumentales que promuevan el análisis crítico, la interpretación y la propuesta de alternativas para superar los problemas que actualmente enfrenta el país. 
12. La Carrera de Trabajo Social cuenta con un plan de estudio y su respectivo perfil profesional, pero requieren ser actualizados, considerando la complejidad de la cuestión social, para que le permita a los nuevos profesionales de la disciplina lograr la vinculación entre la formación teórica y la práctica profesional.

13. La falta de aplicación del modelo educativo de la UNAH evita el desarrollo de procesos de investigación desde el inicio del proceso de formación como parte del quehacer fundamental y de la misma labor docente.

14. Existe desvinculación entre el Colegio de Profesionales de Trabajo Social y la academia, lo que no contribuye con la actualización profesional sistemática de profesionales egresados.

15. El Colegio de Profesionales de Trabajo Social de Honduras no cuenta con un plan estratégico que sirva de marco referencial para la planificación operativa de las diferentes instancias de la estructura organizativa.

16. Una de las mayores debilidades identificada en la estructura y funcionamiento del gremio profesional es el deficiente sistema de comunicación que incide negativamente en el fortalecimiento organizacional y en la identidad profesional.

17. A pesar de la vasta experiencia en las diferentes áreas de intervención profesional, se evidencia la escasa producción de conocimiento científico y de publicaciones por parte de los profesionales agremiados.

18. Se evidencia un vacío en cuanto a la planificación de eventos de carácter científico que posibiliten el encuentro de profesionales a nivel nacional e internacional.

19. En los últimos años, el gremio profesional no ha evidenciado su postura política ante los hechos trascendentales que afectan la soberanía nacional, la democracia, la participación ciudadana y la violación flagrante de los derechos humanos en general.

20. La falta de propuestas y posicionamientos por parte del gremio profesional, ante la realidad nacional, le da una imagen conformista y con poca capacidad de respuesta a las demandas e intereses de sus agremiados y de la población en general.

\section{AGRADECIMIENTOS}

A los alumnos y docentes de la Escuela de Trabajo Social, a la Decanatura de la Facultad de Ciencias Sociales y a la Dirección de Vinculación Universidad-Sociedad por la colaboración prestada. Asimismo, a la Dra. Corina Hernández, directora del Postgrado Latinoamericano de Trabajo Social; a la Msc. Zoila Madrid, docente de la Maestría y al licenciado, Roberto Briceño, jefe del Departamento de Sociología, por la 
asesoría brindada en el desarrollo de esta investigación. De igual manera, gracias a la Dirección de Investigación Científica de la UNAH por proveernos el apoyo financiero necesario para llevar a cabo este estudio.

También gracias a Reina Esperanza Cálix Montes, María Elena Méndez Ordoñez, Duma Midian Valle y María de Jesús Bustillo; quienes colaboraron de alguna manera en el desarrollo de esta investigación.

\section{BIBLIOGRAFÍA}

Carías, Z. M. (2008). Repensando los estudios generales. Tegucigalpa: Editorial Universitaria.

CESPAD. (2011). La profundización del modelo neoliberal en el gobierno actual. Tegucigalpa.

Del Cid García, C. M. (2010). Análisis del contexto, situación política, economica y social de Honduras. Tegucigalpa: Facultad de Economía.

FOSDEH. (2012). Realidad Nacional. Análisis del FOSDEH inicio tercer año administración de Lobo Sosa.

Jorgelina, B. (2007). Desafios a la intervencion profesional de Trabajo Social ante las manifestaciones contemporaneas de la cuestion social. Universidad de Lujan, Buenos Aires, Argentina.

Ludi, M. D. (2003). Formación académica en trabajo social: Una apuesta política para repensar la profesión. Buenos Aires, Argentina.

Molina, M. L. (2010). Estado de Situación de la formacion y organizacion profesional en trabajo social en el contexto de la región México,Centro América y el Caribe. Costa Rica: ALAEITS.

Picado E., M. (2004). Algunos planteamientos para Repensar la Formación profesional en Trabajo Social. En Molina M.,C; Ma. Lorena. La cuestión social y la formación profesional en trrabajo social en el contexto de las nuevas relaciones de poder y la diversidad latinoamericana. San José: ESPACIO Editorial.

Rosas Pagaza, M. (2004). Tendencias teórico-epistemológicas y metodológicas en la formación profesional. XVIII Seminario Latinoamericano de Escuelas de Trabajo Social. Buenos Aires, Argentina.

Tibaná Ríos, D. y. (2009). Fundamentación de la Intervención de Trabajo Social: Sistema conceptual y avances. Bogotá, Colombia.

UNAH, C. d. (1999). Plan de Estudios del Grado de Licenciatura en Trabajo Social. Tegucigalpa.

UNAH, U. N. (2009). Modelo Educativo de la UNAH. Tegucigalpa. 


\section{ANEXOS}

Grupo focal con profesionales de Trabajo Social de la regional en la ciudad de San Pedro Sula.

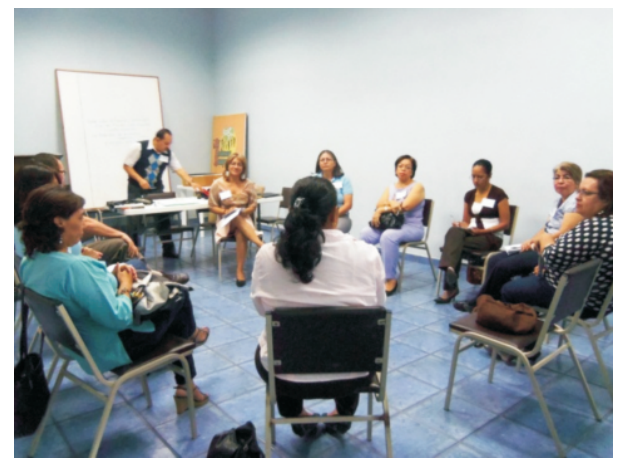

Grupo focal con profesionales de Trabajo Social de la regional en la ciudad de San Pedro Sula

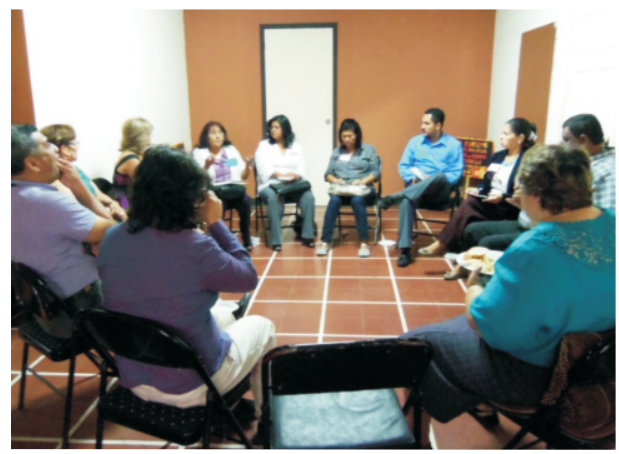

Grupo focal con directivos y ex directivos del Colegio de Profesionales de Trabajo Social

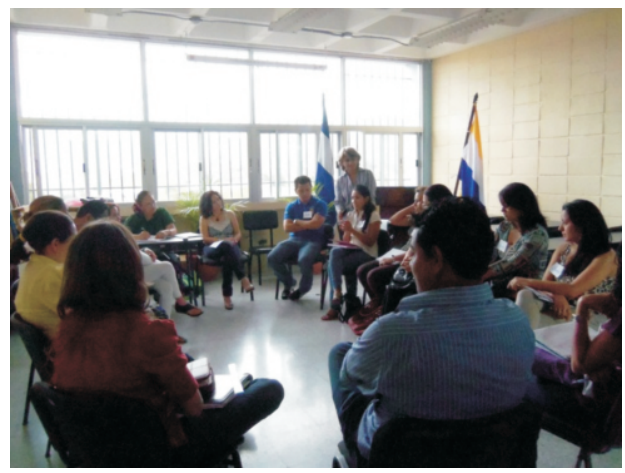

Grupo focal con estudiantes de la Carrera de Trabajo Social - UNAH

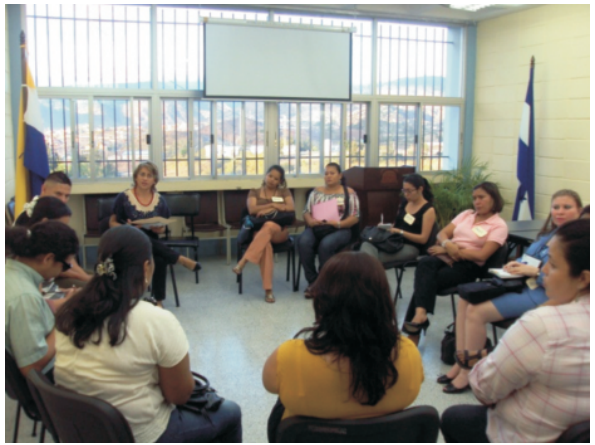

Grupo focal con profesionales de Trabajo Social de la regional en la ciudad de Tegucigalpa

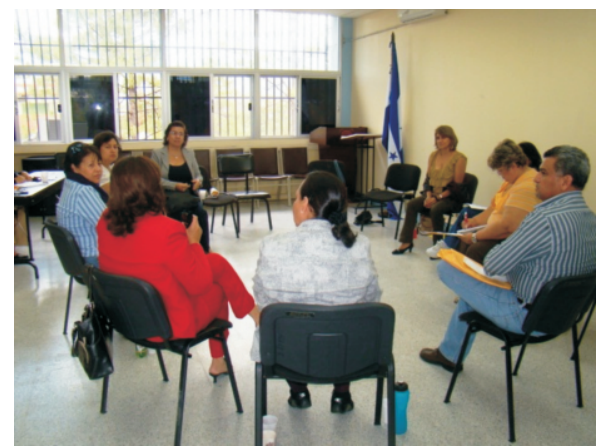

Grupo focal con personal docente de la Carrera de Trabajo Social - UNAH

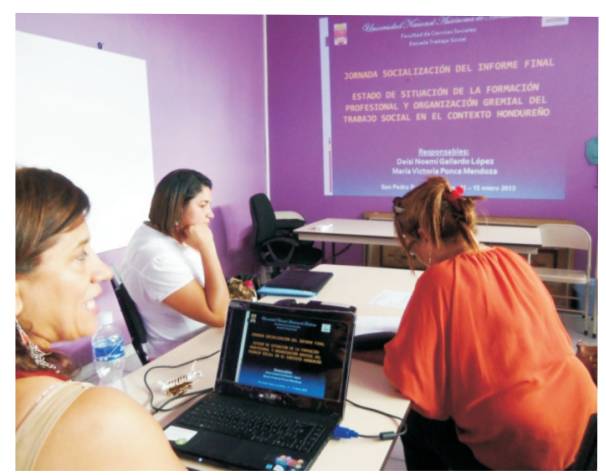

Socialización y validación con profesionales Trabajo Social regional La Ceiba 


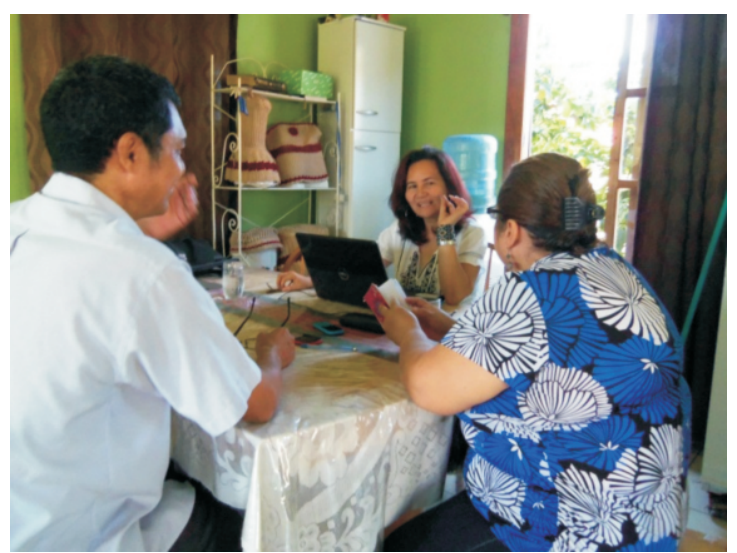

Socialización y validación con profesionales Trabajo Social regional San Pedro Sula

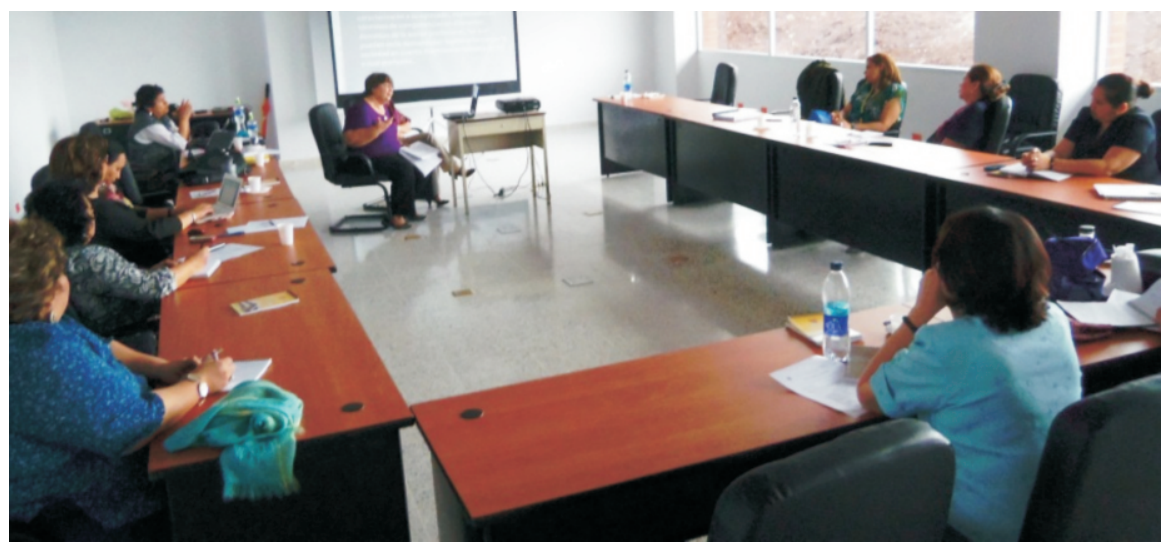

Socialización borrador primer informe de investigación con personal docente de la Carrera de Trabajo Social 